\title{
Passivity-Based Adaptive Control of a 5-DOF Tower Crane
}

\author{
Julia Schatz
}

Submitted under the supervision of Dr. Ryan Caverly to the University Honors Program at the University of Minnesota-Twin Cities in partial fulfillment of the requirements for the degree of Bachelor of Science, magna cum laude in Electrical Engineering.

May 08, 2021 


\begin{abstract}
Tower cranes are often used in construction to transport and lift heavy loads. They are typically controlled by human operators, and the speed, efficacy, and overall cost of their operation could be improved by automation. However, this is a challenging task due to the fact that the system is underactuated and highly nonlinear, which has limited the development of practical dynamic models and control methods of tower cranes. This project contributes to that goal with the derivation of a nonlinear dynamic model and adaptive control method that requiring little knowledge of the system parameters for precise and robust reference tracking. An adaptive control input is derived that ensures the tower crane features a passive input-output mapping. A novel approach is developed to bound the time derivative of the system's mass matrix, which is a critical part of the proof of passivity. Robust closed-loop input-output stability is proven using the Passivity Theorem. Experimental tests are performed, showing the effectiveness of the control law on the threedimensional tower crane.
\end{abstract}

\title{
Non-Technical Summary
}

Tower cranes often used in construction present a challenging control problem. This is because they have parts that are not directly controlled by actuators (the payload sway) and because they have a great number of forces that change based on the position that the crane is in and how it is moving. Typical approaches to control of simpler systems can therefore not be applied. This thesis presents a more advanced control algorithm that continuously estimates the forces on the crane system so that it can more closely follow a desired path. This has the secondary advantage of not requiring any knowledge of the mass or any other particular parameters of the system. The combination of this adaptive control law and a simple feedback control law makes the overall control law mathematically guaranteed to bring the tracking error to zero and remove error in the payload sway. Experimental results are also provided showing the effectiveness of this control law at tracking a path and reducing sway angle compared to a state-of-the-art method.

\section{Contents}

\begin{tabular}{lll}
\hline & Introduction & 1
\end{tabular}

\begin{tabular}{lll}
\hline 2 & Preliminaries & 3
\end{tabular} 
3 System Dynamics 4

3.1 Reformulated Dynamics . . . . . . . . . . . . . . . . . . . . . 6

$\begin{array}{lll}4 \text { Control Formulation } & 7\end{array}$

4.1 Modified System Output . . . . . . . . . . . . . . . . . . . . . . . . . 8

4.2 Adaptive Control Law . . . . . . . . . . . . . . . . . . . . . . . . . . . . . . . . . . . 8

4.3 Error Dynamics . . . . . . . . . . . . . . . . . . . . . . . . . . . . . . . . . . . 10

4.4 Passivity and Closed-Loop Stability Analysis . . . . . . . . . . . . . . 11

4.5 Selection of Bounding Parameter . . . . . . . . . . . . . . . . . . . . 18

$\begin{array}{lll}5 & \text { Numerical Results } & 21\end{array}$

$\begin{array}{lll}6 & \text { Experimental Results } & 22\end{array}$

\begin{tabular}{lll}
\hline 7 & Conclusion & 29
\end{tabular}

\begin{tabular}{|l|}
\hline A Mass Matrix and Nonlinear Force Terms \\
\hline 3
\end{tabular}

\section{List of Figures}

$1 \quad$ Negative feedback interconnection of OSP systems. . . . . . . . . . . 4

2 (a) Isometric view of the tower crane showing jib rotation, (b) Side view showing the cart movement and radial sway angle, and (c) Front view showing the tangential sway angle. . . . . . . . . . . . . 5

$3 \quad$ Block diagram showing passive interconnection of controllers. . . . . . 14

4 Maximum real eigenvalue part of the linearized closed-loop system . . 23

$5 \quad$ Picture of the Quanser tower crane used in section 6. . . . . . . . . . 25

$6 \quad$ Payload positions over time with both controllers. . . . . . . . . . . . 27

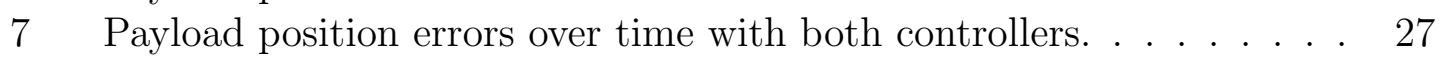

$8 \quad$ Payload velocity errors over time with both controllers. . . . . . . . . 28

$9 \quad$ SPR controller forces over time. . . . . . . . . . . . . . . . . . . . . . 28

$10 \quad$ Constant gain controller forces over time. . . . . . . . . . . . . . . . . . 29 


\section{Introduction}

Tower cranes are often found on construction sites, providing a simple solution to lifting heavy loads to high places. However, their complexity of operation and reliance on highly-skilled human operators makes them prime candidates for automation. Unfortunately, tower cranes are underactuated and dominated by nonlinear dynamics, making for a challenging control problem.

Autonomous tower crane payload trajectory tracking control is not an entirely new concept. A number of control methods have been proposed over the last couple of decades to solve this challenging problem. Some of the most well-studied techniques take advantage of open-loop input shaping to control payload sway [1 3$]$. Practical closed-loop control techniques have also been developed for tower crane, including adaptive integral sliding-mode control [4], nonlinear model predictive control [5], gain-scheduled control [6], adaptive control [7, 8], and passivity-based control [9]. Many of these control techniques have distinct limitations. Some [5, 7] rely on a specific model formulation and are thus potentially sensitive to unmodeled dynamics. Others [1-3] do not provide an end-to-end control solution and tend to rely on linearization or exact knowledge of the system parameters. Therefore, they can be ineffective in practice outside of perturbations near the linearization point. Additionally, very few of these approaches account for all five degrees of freedom of the tower crane dynamics.

Passivity analysis has been widely used in control of robotic manipulators $9-13$. It differs from Lyapunov-based analysis by focusing on the input-output properties of the system and provides a method to prove closed-loop input-output stability, rather 
than closed-loop asymptotic stability where the states converge to zero asymptotically. The Passivity Theorem [14] can be used to determine suitable classes of feedback controllers that ensure closed-loop input-output stability for a plant with a given passivity property. This framework allows for simple analysis and flexibility in the design and selection of a feedback controller, as an entire class of feedback controllers is determined to be stabilizing via the Passivity Theorem. Moreover, it can often be shown that a plant retains its passivity property in the presence of large amounts of model uncertainty, which allows for robust input-output stability to be guaranteed by the Passivity Theorem.

The work of [9] was the first to make use of passivity-based control for the payload trajectory tracking of a two -dimensional tower crane with a flexible hoist cable. Although this method showed promising results, it relied on the restrictive assumptions that the system's dynamics were known exactly and that the payload sway angle and sway angle rates remain small. Preliminary work on a passivity-based adaptive control method that removes these assumptions was performed in [15] for a two-dimensional tower crane. This thesis substantially extends the preliminary work in [15] for use with a 5 degree-of-freedom (DOF) tower crane in three dimensions. The novel contributions specific to this thesis include (1) a passivity-based adaptive payload trajectory tracking control law that fully accounts for the nonlinear dynamics of a 5-DOF tower crane and (2) a convex optimization approach to determine robust bounds on the time derivative of the system's mass matrix, which are needed to ensure the system has a passive input-output mapping. The proposed control law is also among the first to make use of all five degrees of freedom of a three-dimensional 
tower crane. The proposed control law does not require exact knowledge of the system parameters, does not depend on the exact structure of the dynamic model, and provides a mathematically rigorous and provably input-output stable end-to-end trajectory tracking control solution. In addition, the passivity-based control formulation employed in this work guarantees closed-loop input-output stability with any output strictly passive (OSP) feedback controller.

The format of the remainder of this thesis is as follows. The dynamic model of the system is briefly discussed and reformulated into a convenient form in Section 3 . In Section 4, the control law is formulated through the definition of a modified system output, an adaptive control law, and an OSP feedback controller. Numerical linearization results are included in Section 5 and physical experimental results are given in Section 6. Concluding remarks are given in Section 7 .

\section{Preliminaries}

Theorems, concepts, and important notation that are foundational to the technical approaches used in this thesis are provided in this section.

The positive definiteness of a matrix is denoted in this thesis by $>0$. The identity matrix is written as $\mathbf{1}$ and a matrix of zeros is written as $\mathbf{0}$. The signal $\mathbf{y}(t)$ is an element of the Lebesgue space $\mathcal{L}_{2}$, that is $\mathbf{y} \in \mathcal{L}_{2}$, if $\|\mathbf{y}\|_{2}^{2}=\int_{0}^{\infty} \mathbf{y}^{\top}(t) \mathbf{y}(t) \mathrm{d} t<$ $\infty$. Similarly, the signal $\mathbf{y}(t)$ is an element of the extended Lebesgue space $\mathcal{L}_{2 e}$, that is $\mathbf{y} \in \mathcal{L}_{2 e}$, if $\|\mathbf{y}\|_{2 T}^{2}=\int_{0}^{\infty} \mathbf{y}_{T}^{\top}(t) \mathbf{y}_{T}(t) \mathrm{d} t<\infty, T \in \mathbb{R}_{\geq 0}$, where $\mathbf{y}_{T}(t)=\mathbf{y}(t)$ for $0 \leq t \leq T$ and $\mathbf{y}_{T}(t)=\mathbf{0}$ for $T<t[16]$. Additionally, $\mathbf{y} \in \mathcal{L}_{\infty}$ if $\|\mathbf{y}\|_{\infty}=$ 


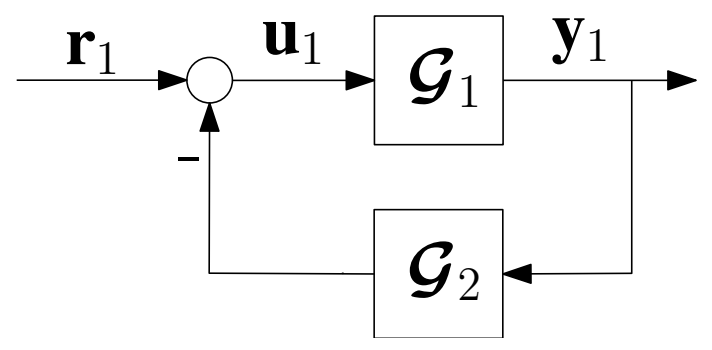

Figure 1: Negative feedback interconnection of OSP systems.

$\sup _{t \in \mathbb{R}_{\geq 0}}\left[\max _{i=1, \ldots, n}\left|y_{i}(t)\right|\right]<\infty$, where $\mathbf{y}^{\top}(t)=\left[\begin{array}{lll}y_{1}(t) & \cdots & \left.y_{n}(t)\right]\end{array}\right.$

Definition 1 (Passivity [16]). The input-output mapping $\mathbf{u} \mapsto \mathbf{y}$ associated with the operator $\mathcal{G}: \mathcal{L}_{2 e} \rightarrow \mathcal{L}_{2 e}$, where $\mathbf{y}=\mathcal{G}(\mathbf{u})$, is output strictly passive (OSP) if there exist constants $\in \in \mathbb{R}_{>0}$ and $\beta \in \mathbb{R}$ such that

$$
\int_{0}^{T} \mathbf{y}^{\top}(t) \mathbf{u}(t) \mathrm{d} t \geq \epsilon\|\mathbf{y}\|_{2 T}^{2}+\beta
$$

If (1) is satisfied with $\epsilon=0$, then $\mathbf{u} \mapsto \mathbf{y}$ is passive. The scalar $\beta$ is a constant related to initial conditions.

Theorem 1 (Passivity Theorem [16]). Consider the negative feedback interconnection of $\mathcal{G}_{1}: \mathcal{L}_{2 e} \rightarrow \mathcal{L}_{2 e}$ and $\mathcal{G}_{2}: \mathcal{L}_{2 e} \rightarrow \mathcal{L}_{2 e}$, defined as $\mathbf{u}_{1}=\mathbf{r}_{1}-\mathcal{G}_{2}\left(\mathbf{y}_{1}\right), \mathbf{y}_{1}=\mathcal{G}_{1}\left(\mathbf{u}_{1}\right)$ where $\mathbf{r}_{1}$ is a reference signal. If $\mathcal{G}_{1}$ and $\mathcal{G}_{2}$ are both $O S P$, then $\mathbf{y}_{1}, \mathbf{u}_{1} \in \mathcal{L}_{2}$ for every $\mathbf{r}_{1} \in \mathcal{L}_{2}$

\section{System Dynamics}

Consider the three-dimensional tower crane depicted in Figure 2. The jib cart is modeled as point mass $m_{1}$, located at point $y_{1}$ and at distance $x$ from the fixed point 


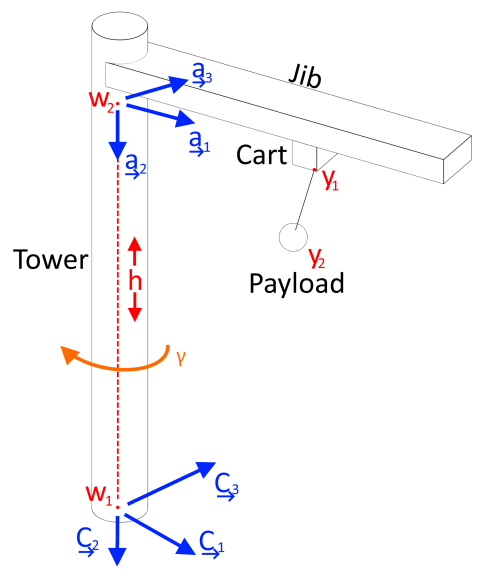

(a)

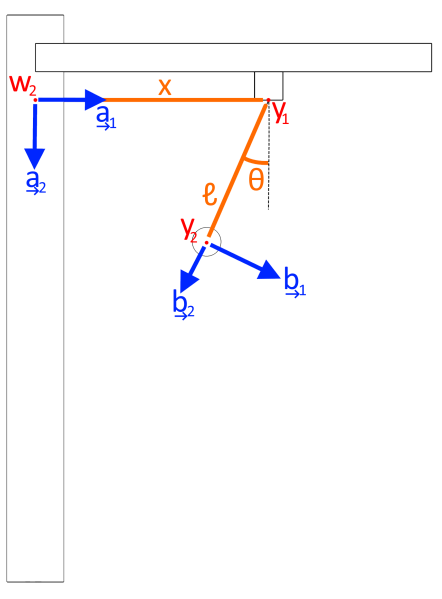

(b)

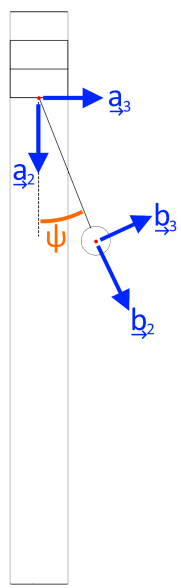

(c)

Figure 2: (a) Isometric view of the tower crane showing jib rotation, (b) Side view showing the cart movement and radial sway angle, and (c) Front view showing the tangential sway angle.

$w_{2}$. This cart is the attachment point of the payload hoist cable, which has length $\ell$ and connects to a point-mass payload $m_{2}$ located at point $y_{2}$. To simplify notation, the position of $y_{2}$ relative to $w_{1}$ in frame $\mathcal{F}_{c}$ is labeled $\boldsymbol{\rho}$ in the remainder of this thesis. The overall height of the crane is the distance between the points $w_{1}$ and $w_{2}$, and is labeled $h$. The angle between the inertial reference frame $\mathcal{F}_{c}$ and the jib frame $\mathcal{F}_{a}$ is $\gamma$ about $\underline{c}_{2}$. The radial sway angle $\theta$ of the hoist cable is about $\underline{a}_{3}$. The tangential sway angle $\psi$ of the hoist cable is about $b_{1}$. The reference frame $\mathcal{F}_{b}$ rotates with the hoist cable, where $\underline{b}_{2}$ points in the direction of the cable. The rotation from $\mathcal{F}_{a}$ to $\mathcal{F}_{b}$ is defined by a 3-1 Euler angle sequence in the angles $\theta$ and $\psi$. To simplify the model, the hoist cable is assumed to be rigid. The generalized coordinates of the tower crane are summarized as $\mathbf{q}^{\top}=\left[\begin{array}{lllll}x & \ell & \gamma & \theta & \psi\end{array}\right]$, all of which are assumed to be quantities that can be measured.

The tower crane is actuated by a force applied in the $a_{\rightarrow}$ direction to the jib cart, 
$f_{x}$, a tensile force applied to the hoist cable in the $\underline{b}_{2}$ direction, $f_{\ell}$, and a torque applied to the tower about $\stackrel{c}{\rightarrow}_{2}, \tau_{\gamma}$. The equations of motion of the tower crane are $4,7,8,15$

$$
\mathbf{M} \ddot{\mathbf{q}}+\mathbf{D} \dot{\mathbf{q}}=\mathbf{f}+\mathbf{f}_{\text {non }}(\mathbf{q}, \dot{\mathbf{q}})
$$

where $\mathbf{M}(\mathbf{q})=\mathbf{M}^{\top}(\mathbf{q})>0$ is the mass matrix, $\mathbf{D}=\operatorname{diag}\left\{0,0,0, c_{\theta}, c_{\psi}\right\}$ is the damping matrix that accounts for natural damping of the hoist cable sway with $c_{\theta}, c_{\psi} \in \mathbb{R}_{\geq 0}$, $\mathbf{f}^{\top}=\left[\begin{array}{lll}\hat{\mathbf{f}}^{\top} & 0 & 0\end{array}\right]=\left[\begin{array}{lllll}f_{x} & f_{\ell} & \tau_{\gamma} & 0 & 0\end{array}\right]$ contains the actuated inputs to the crane, and $f_{\text {non }}(\mathbf{q}, \dot{\mathbf{q}})=\mathbf{G}_{\text {non }}(\mathbf{q})+\mathbf{C}_{\text {non }}(\mathbf{q}, \dot{\mathbf{q}}) \dot{\mathbf{q}}$ contains nonlinear terms, including gravitational and centrifugal forces. Further details on the contents of the mass matrix and the nonlinear terms are given in Appendix A.

\subsection{Reformulated Dynamics}

The tower crane's equations of motion are reformulated in this section into a form that partitions the actuated $\left(\mathbf{q}_{a}^{\top}=\left[\begin{array}{lll}x & \ell & \gamma\end{array}\right]\right)$ and unactuated $\left(\mathbf{q}_{u}^{\top}=\left[\begin{array}{ll}\theta & \psi\end{array}\right]\right)$ terms. The equations of motion in (2) are rewritten as

$$
\begin{gathered}
\mathbf{M}_{a a} \ddot{\mathbf{q}}_{a}+\mathbf{M}_{a u} \ddot{\mathbf{q}}_{u}=\hat{\mathbf{f}}+\mathbf{C}_{a a} \dot{\mathbf{q}}_{a}+\mathbf{C}_{a u} \dot{\mathbf{q}}_{u}+\mathbf{G}_{a}, \\
\mathbf{M}_{u u} \ddot{\mathbf{q}}_{u}+\mathbf{M}_{u a} \ddot{\mathbf{q}}_{a}+\mathbf{D}_{u} \dot{\mathbf{q}}_{u}=\mathbf{C}_{u u} \dot{\mathbf{q}}_{u}+\mathbf{C}_{u a} \dot{\mathbf{q}}_{a}+\mathbf{G}_{u},
\end{gathered}
$$


where

$$
\begin{gathered}
\mathbf{M}=\left[\begin{array}{ll}
\mathbf{M}_{a a} & \mathbf{M}_{a u} \\
\mathbf{M}_{u a} & \mathbf{M}_{u u}
\end{array}\right], \quad \mathbf{C}=\left[\begin{array}{ll}
\mathbf{C}_{a a} & \mathbf{C}_{a u} \\
\mathbf{C}_{u a} & \mathbf{C}_{u u}
\end{array}\right], \\
\mathbf{G}=\left[\begin{array}{l}
\mathbf{G}_{a} \\
\mathbf{G}_{u}
\end{array}\right], \quad \mathbf{D}=\left[\begin{array}{cc}
\mathbf{0} & \mathbf{0} \\
\mathbf{0} & \mathbf{D}_{u}
\end{array}\right]
\end{gathered}
$$

and we note that $\mathbf{M}_{a u}=\mathbf{M}_{u a}^{\top}$.

Isolating for $\ddot{\mathbf{q}}_{u}$ in (4) and combining the resulting equation with (3) gives the reformulated equations of motion

$$
\begin{gathered}
\ddot{\mathbf{q}}_{u}=\mathbf{M}_{u u}^{-1}\left(-\mathbf{M}_{u a} \ddot{\mathbf{q}}_{a}-\mathbf{D}_{u} \dot{\mathbf{q}}_{u}+\mathbf{C}_{u u} \dot{\mathbf{q}}_{u}+\mathbf{C}_{u a} \dot{\mathbf{q}}_{a}+\mathbf{G}_{u}\right), \\
\overline{\mathbf{M}} \ddot{\mathbf{q}}_{a}+\overline{\mathbf{C}}_{a} \dot{\mathbf{q}}_{a}+\overline{\mathbf{C}}_{u} \dot{\mathbf{q}}_{u}+\overline{\mathbf{G}}=\hat{\mathbf{f}}
\end{gathered}
$$

where $\overline{\mathbf{M}}=\mathbf{M}_{a a}-\mathbf{M}_{a u} \mathbf{M}_{u u}^{-1} \mathbf{M}_{u a}, \overline{\mathbf{C}}_{a}=\mathbf{M}_{a u} \mathbf{M}_{u u}^{-1} \mathbf{C}_{u a}-\mathbf{C}_{a a}, \overline{\mathbf{C}}_{u}=\mathbf{M}_{a u} \mathbf{M}_{u u}^{-1}\left(\mathbf{C}_{u u}+\right.$ $\left.\mathbf{D}_{u}\right)-\mathbf{C}_{a u}$, and $\overline{\mathbf{G}}=\mathbf{M}_{a u} \mathbf{M}_{u u}^{-1} \mathbf{G}_{u}-\mathbf{G}_{a}$. Note that the positive definiteness of the matrices $\overline{\mathbf{M}}$ and $\mathbf{M}_{u u}$ is guaranteed by the positive definiteness of $\mathbf{M}$ through the Schur complement lemma 17 .

\section{Control Formulation}

The formulation of the proposed control law proceeds by first presenting the modified system output. An adaptive control law based on this modified output is then introduced and shown to yield an OSP input-output mapping in the tower crane system. Two OSP feedback controllers are proposed and stability of the closed-loop system is analyzed. 


\subsection{Modified System Output}

Inspired by [15, 18, 19], a modified system output, $\mathbf{s}$, is defined as

$$
\mathbf{s}=\dot{\tilde{\mathbf{q}}}_{a}+\lambda \tilde{\mathbf{q}}_{a}+\boldsymbol{\alpha} \mathbf{q}_{u}
$$

where $\tilde{\mathbf{q}}_{a}=\mathbf{q}_{a}-\mathbf{q}_{a, d}$ and $\dot{\tilde{\mathbf{q}}}_{a}=\dot{\mathbf{q}}_{a}-\dot{\mathbf{q}}_{a, d}$ are the payload position and velocity tracking errors, $\mathbf{q}_{a, d}$ and $\dot{\mathbf{q}}_{a, d}$ are the desired payload position and velocity, and $\boldsymbol{\lambda}_{\rho}=\operatorname{diag}\left\{\lambda_{1}, \lambda_{2}, \lambda_{3}\right\}$ and $\boldsymbol{\alpha}=\left[\begin{array}{cc}\alpha_{1} & 0 \\ 0 & 0 \\ 0 & \alpha_{2}\end{array}\right]$ are constant control parameters satisfying $\lambda_{1}, \lambda_{2}, \lambda_{3}, \alpha_{1}, \alpha_{2} \in \mathbb{R}_{\geq 0}$. The time derivative of $\mathbf{s}$ is

$$
\dot{\mathbf{s}}=\ddot{\tilde{\mathbf{q}}}_{a}+\lambda \dot{\tilde{\mathbf{q}}}_{a}+\boldsymbol{\alpha} \dot{\mathbf{q}}_{u}
$$

where $\ddot{\tilde{q}}_{a}=\ddot{\mathbf{q}}_{a}-\ddot{\mathbf{q}}_{a, d}$ is the payload acceleration tracking error and $\ddot{\mathbf{q}}_{a, d}$ is the desired payload acceleration. Combining (7) and (8) gives

$$
\dot{\mathbf{s}}+\boldsymbol{\lambda} \mathbf{s}=\ddot{\tilde{\mathbf{q}}}_{a}+2 \boldsymbol{\lambda} \dot{\tilde{\mathbf{q}}}_{a}+\boldsymbol{\lambda}^{\top} \boldsymbol{\lambda} \tilde{\mathbf{q}}_{a}+\boldsymbol{\alpha} \dot{\mathbf{q}}_{u}+\boldsymbol{\lambda} \boldsymbol{\alpha} \mathbf{q}_{u} .
$$

The reference acceleration is defined using $(9)$ as

$$
\ddot{\mathbf{q}}_{a, r}=\ddot{\mathbf{q}}_{a}-(\dot{\mathbf{s}}+\lambda \mathbf{s}) .
$$

\subsection{Adaptive Control Law}

Inspired by the work of [10,12,20], an adaptive control law is chosen that acts as a feedforward-like control input through an estimate of the system dynamics. To 
obtain the form of the adaptive control input, the reformulated equations of motion from (5) and (6), are factored into the form $\mathbf{u}=\mathbf{W a}$, where $\mathbf{a}^{\top}=\left[\begin{array}{llll}\overline{\mathbf{m}}^{\top} & \overline{\mathbf{c}}_{a}^{\top} & \overline{\mathbf{c}}_{u}^{\top} & \overline{\mathbf{g}}^{\top}\end{array}\right]$,

$$
\mathbf{W}=\left[\begin{array}{cccccccccccc}
\ddot{\mathbf{q}}_{a, r}^{\top} & \mathbf{0} & \mathbf{0} & \dot{\mathbf{q}}_{a}^{\top} & \mathbf{0} & \mathbf{0} & \dot{\mathbf{q}}_{u}^{\top} & \mathbf{0} & \mathbf{0} & 1 & 0 & 0 \\
\mathbf{0} & \ddot{\mathbf{q}}_{a, r}^{\top} & \mathbf{0} & \mathbf{0} & \dot{\mathbf{q}}_{a}^{\top} & \mathbf{0} & \mathbf{0} & \dot{\mathbf{q}}_{u}^{\top} & \mathbf{0} & 0 & 1 & 0 \\
\mathbf{0} & \mathbf{0} & \dot{\mathbf{q}}_{a, r}^{\top} & \mathbf{0} & \mathbf{0} & \dot{\mathbf{q}}_{a}^{\top} & \mathbf{0} & \mathbf{0} & \dot{\mathbf{q}}_{u}^{\top} & 0 & 0 & 1
\end{array}\right]
$$

$\mathbf{u}=\hat{\mathbf{f}}, \ddot{\mathbf{q}}_{a}$ is replaced with the reference acceleration 100 , and the entries of a correspond to the columnized versions of the matrices $\overline{\mathbf{M}}, \overline{\mathbf{C}}_{a}, \overline{\mathbf{C}}_{u}$, and $\overline{\mathbf{G}}$ in (5) and (6). The matrix $\mathbf{W}$ contains a subset of the system states that are assumed to be measureable. In practice, the parameters of the system dynamics contained in a will not be precisely known. Instead, an estimate, $\hat{\mathbf{a}}$, is used that satisfies

$$
\mathbf{W} \hat{\mathbf{a}}=\hat{\mathbf{M}} \ddot{\mathbf{q}}_{a, r}+\hat{\mathbf{C}}_{a} \dot{\mathbf{q}}_{a}+\hat{\mathbf{C}}_{u} \dot{\mathbf{q}}_{u}+\hat{\mathbf{G}}
$$

The adaptive control input is then given by

$$
\hat{\mathbf{u}}=\mathbf{W} \hat{\mathbf{a}}-\left(\frac{1}{2} \dot{\mathbf{q}}^{\top} \boldsymbol{\delta}\right) \mathbf{s}
$$

where the column matrix $\boldsymbol{\delta}$ is chosen such that

$$
\dot{\overline{\mathbf{M}}}(\mathbf{q}, \dot{\mathbf{q}})-\left(\dot{\mathbf{q}}^{\top} \boldsymbol{\delta}\right) \mathbf{1}<0
$$


The selection of $\boldsymbol{\delta}^{\top}=\left[\begin{array}{lllll}\delta_{x} & \delta_{\ell} & \delta_{\gamma} & \delta_{\theta} & \delta_{\psi}\end{array}\right]$ is discussed in more detail in Section 4.5 . Following the approach in [10,12, 20, the adaptive update law is chosen as

$$
\dot{\hat{\mathbf{a}}}=-\Gamma^{\top} \mathbf{W}^{\top} \mathbf{s},
$$

where $\boldsymbol{\Gamma}=\boldsymbol{\Gamma}^{\boldsymbol{\top}}>0$ is the tuning parameter of the update law.

The complete control law is given by

$$
\mathbf{u}=\hat{\mathbf{u}}+\overline{\mathbf{u}}
$$

where $\overline{\mathbf{u}}$ is some feedback control input and $\hat{\mathbf{u}}$ is the adaptive control input from (12).

\subsection{Error Dynamics}

Substituting the control law of 15 with the adaptive control law of (11) into the reformulated equations of motion in (5) and (6) gives

$$
\overline{\mathbf{M}} \ddot{\mathbf{q}}_{a}=\hat{\mathbf{M}} \ddot{\mathbf{q}}_{a, r}+\tilde{\mathbf{C}}_{a} \dot{\mathbf{q}}_{a}+\tilde{\mathbf{C}}_{u} \dot{\mathbf{q}}_{u}+\tilde{\mathbf{G}}-\left(\frac{1}{2} \dot{\mathbf{q}}^{\top} \boldsymbol{\delta}\right) \mathbf{s}+\overline{\mathbf{u}},
$$

where $\tilde{\mathbf{C}}_{a}=\hat{\mathbf{C}}_{a}-\overline{\mathbf{C}}_{a}, \tilde{\mathbf{C}}_{u}=\hat{\mathbf{C}}_{u}-\overline{\mathbf{C}}_{u}$, and $\tilde{\mathbf{G}}=\hat{\mathbf{G}}-\overline{\mathbf{G}}$. Subtracting $\overline{\mathbf{M}} \ddot{\mathbf{q}}_{a, r}$ from both sides of (16) yields

$$
\overline{\mathbf{M}}\left(\ddot{\mathbf{q}}_{a}-\ddot{\mathbf{q}}_{a, r}\right)=\tilde{\mathbf{M}} \ddot{\mathbf{q}}_{a, r}+\tilde{\mathbf{C}}_{a} \dot{\mathbf{q}}_{a}+\tilde{\mathbf{C}}_{u} \dot{\mathbf{q}}_{u}+\tilde{\mathbf{G}}-\left(\frac{1}{2} \dot{\mathbf{q}}^{\top} \boldsymbol{\delta}\right) \mathbf{s}+\overline{\mathbf{u}}
$$


Substituting (10) into (17) and rearranging results in

$$
\overline{\mathbf{M}} \dot{\mathbf{s}}=\tilde{\mathbf{M}} \ddot{\mathbf{q}}_{a, r}+\tilde{\mathbf{C}}_{a} \dot{\mathbf{q}}_{a}+\tilde{\mathbf{C}}_{u} \dot{\mathbf{q}}_{u}+\tilde{\mathbf{G}}-\left(\frac{1}{2} \dot{\mathbf{q}}^{\top} \boldsymbol{\delta}\right) \mathbf{s}-\overline{\mathbf{M}} \boldsymbol{\lambda} \mathbf{s}+\overline{\mathbf{u}}
$$

The error dynamics in $(18)$ can be rewritten as

$$
\overline{\mathbf{M}} \dot{\mathbf{s}}=\mathbf{W} \tilde{\mathbf{a}}-\left(\frac{1}{2} \dot{\mathbf{q}}^{\top} \boldsymbol{\delta}\right) \mathbf{s}-\overline{\mathbf{M}} \boldsymbol{\lambda} \mathbf{s}+\overline{\mathbf{u}}
$$

where

$$
\mathbf{W} \tilde{\mathbf{a}}=\mathbf{W}(\hat{\mathbf{a}}-\mathbf{a})=\tilde{\mathbf{M}} \ddot{\mathbf{q}}_{a, r}+\tilde{\mathbf{C}}_{a} \dot{\mathbf{q}}_{a}+\tilde{\mathbf{C}}_{u} \dot{\mathbf{q}}_{u}+\tilde{\mathbf{G}}
$$

\subsection{Passivity and Closed-Loop Stability Analysis}

The following theorems demonstrate that the tower crane system features an OSP input-output mapping and analyze the closed-loop stability properties of the system with two proposed OSP feedback controllers.

Theorem 2. Consider the tower crane system with error dynamics defined in (19), where the term $\hat{\mathbf{a}}$ is updated based on the adaptive law in (14) and $\mathbf{a}$ is assumed to vary slowly in time. The input-output mapping $\overline{\mathbf{u}} \mapsto \mathbf{s}$ is OSP.

Proof. Consider the function

$$
V=\frac{1}{2} \mathbf{s}^{\top} \overline{\mathbf{M}} \mathbf{s}+\frac{1}{2} \tilde{\mathbf{a}}^{\top} \boldsymbol{\Gamma}^{-1} \tilde{\mathbf{a}}
$$

The matrices $\overline{\mathbf{M}}$ and $\boldsymbol{\Gamma}$ are positive definite, which ensures that $V$ is nonnegative. 
Taking the time derivative of (21) and substituting in $(19)$ gives

$$
\begin{aligned}
& \dot{V}=\mathbf{s}^{\top} \overline{\mathbf{M}} \dot{\mathbf{s}}+\frac{1}{2} \mathbf{s}^{\top} \dot{\overline{\mathbf{M}}} \mathbf{s}+\dot{\tilde{\mathbf{a}}}^{\top} \boldsymbol{\Gamma}^{-1} \tilde{\mathbf{a}} \\
& =\mathbf{s}^{\top}\left(\mathbf{W} \tilde{\mathbf{a}}-\left(\frac{1}{2} \dot{\mathbf{q}}^{\top} \boldsymbol{\delta}\right) \mathbf{s}-\overline{\mathbf{M}} \boldsymbol{\lambda} \mathbf{s}+\overline{\mathbf{u}}\right) \\
& +\frac{1}{2} \mathbf{S}^{\top} \dot{\overline{\mathbf{M}}} \mathbf{s}+\dot{\tilde{\mathbf{a}}}^{\top} \boldsymbol{\Gamma}^{-1} \tilde{\mathbf{a}} \\
& =\mathbf{S}^{\top} \overline{\mathbf{u}}+\frac{1}{2} \mathbf{S}^{\top}\left(\dot{\overline{\mathbf{M}}}-\left(\frac{1}{2} \dot{\mathbf{q}}^{\top} \boldsymbol{\delta}\right)\right) \mathbf{S} \\
& -\mathbf{s}^{\top} \overline{\mathbf{M}} \boldsymbol{\lambda} \mathbf{s}+\left(\mathbf{s}^{\top} \mathbf{W}+\dot{\tilde{\mathbf{a}}}^{\top} \boldsymbol{\Gamma}^{-1}\right) \tilde{\mathbf{a}} .
\end{aligned}
$$

Recalling the matrix inequality in $(13), 22$ can be rewritten as

$$
\dot{V} \leq \mathbf{s}^{\top} \overline{\mathbf{u}}-\mathbf{s}^{\top} \overline{\mathbf{M}} \boldsymbol{\lambda} \mathbf{s}+\left(\mathbf{s}^{\top} \mathbf{W}+\dot{\tilde{\mathbf{a}}}^{\top} \boldsymbol{\Gamma}^{-1}\right) \tilde{\mathbf{a}}
$$

Knowing that a varies slowly in time, it is approximated that $\dot{\tilde{\mathbf{a}}}=\dot{\hat{\mathbf{a}}}-\dot{\mathbf{a}} \approx \dot{\hat{\mathbf{a}}}$, where $\dot{\hat{\mathbf{a}}}=-\boldsymbol{\Gamma}^{\top} \mathbf{W}^{\top} \mathbf{s}$ from the adaptive update law in 114 . Substituting this into 23. results in

$$
\dot{V} \leq \mathbf{s}^{\top} \overline{\mathbf{u}}-\mathbf{s}^{\top} \overline{\mathbf{M}} \boldsymbol{\lambda} \mathbf{s}
$$

Taking the integral of both sides of this inequality from $t=0$ to $t=T \geq 0$ gives

$$
\begin{aligned}
\int_{0}^{T} \dot{V} \mathrm{~d} t & \leq \int_{0}^{T} \mathbf{s}^{\top} \overline{\mathbf{u}} \mathrm{d} t-\int_{0}^{T} \mathbf{s}^{\top} \overline{\mathbf{M}} \boldsymbol{\lambda} \mathbf{s} \mathrm{d} t \\
& \leq \int_{0}^{T} \mathbf{s}^{\top} \overline{\mathbf{u}} \mathrm{d} t-\lambda_{\min }(\overline{\mathbf{M}} \boldsymbol{\lambda})\|\mathbf{s}\|_{2 T}^{2} .
\end{aligned}
$$


Rearranging (25) gives

$$
\int_{0}^{T} \mathbf{s}^{\top} \overline{\mathbf{u}} \mathrm{d} t \geq V(T)-V(0)+\epsilon\|\mathbf{s}\|_{2 T}^{2},
$$

where $\epsilon=\lambda_{\min }(\overline{\mathbf{M}} \boldsymbol{\lambda})>0$ since $\overline{\mathbf{M}} \boldsymbol{\lambda}>0$. Because $V(T) \geq 0$, (26) can be rewritten as

$$
\int_{0}^{T} \mathbf{s}^{\top} \overline{\mathbf{u}} \mathrm{d} t \geq-V(0)+\epsilon\|\mathbf{s}\|_{2 T}^{2}
$$

which proves that the mapping $\overline{\mathbf{u}} \mapsto \mathbf{s}$ is OSP based on Definition 1 .

Since the input-output mapping $\overline{\mathbf{u}} \mapsto \mathbf{s}$ is OSP, from Theorem 1 it is known that any OSP controller in a negative feedback interconnection with this system ensures closed-loop input-output stability, as represented in Figure 3. Note that the OSP input-output mapping proven in Theorem 2 holds regardless of the specific form of the system dynamics and does not require exact knowledge of the system parameters, other than a bound on the time derivative of the mass matrix in (13). This means that robust closed-loop input-output stability is guaranteed, provided an OSP feedback controller is implemented.

Two OSP feedback controllers are examined in this thesis: a constant gain controller and a dynamic strictly positive real (SPR) controller. The constant gain controller is defined by

$$
\overline{\mathbf{u}}=-\mathbf{K s},
$$

where $\mathbf{K}=\mathbf{K}^{\boldsymbol{\top}}>0$ is the control gain. 


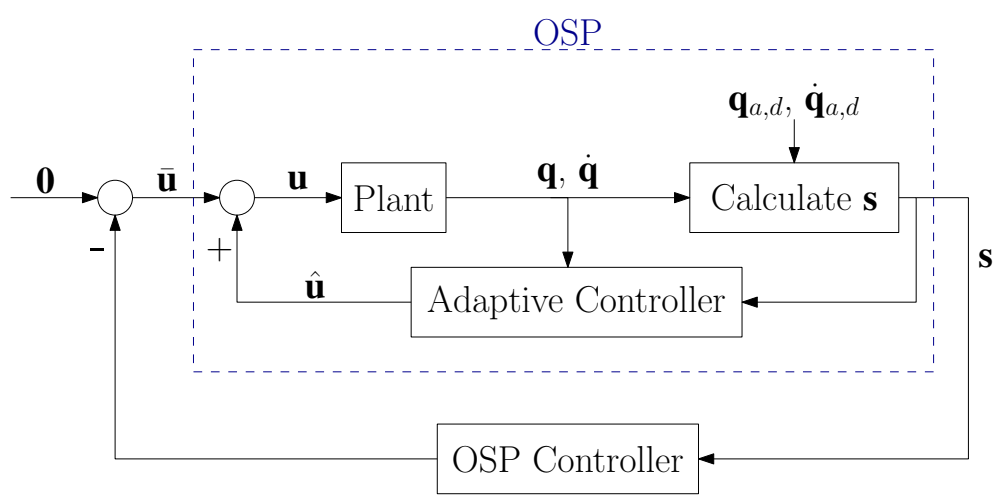

Figure 3: Block diagram showing passive interconnection of controllers.

Theorem 3. Consider the tower crane system described by the equations of motion in (5) and (6). The control law defined by (15) and (28) ensures that $\mathbf{s} \in \mathcal{L}_{2} \cap \mathcal{L}_{\infty}$.

Proof. Consider the function $V$ from 21. Following the same procedure used in the proof of Theorem 2, substituting the control law $\overline{\mathbf{u}}=-\mathbf{K s}$ into (24), and knowing $\overline{\mathbf{M}} \boldsymbol{\lambda}>0$ results in

$$
\dot{V} \leq-\mathbf{s}^{\top} \mathbf{K} \mathbf{s}-\mathbf{s}^{\top} \overline{\mathbf{M}} \boldsymbol{\lambda} \mathbf{s} \leq-\mathbf{s}^{\top} \mathbf{K} \mathbf{s} \leq 0
$$

Knowing that $V(T) \geq 0$, this result shows that $V(T) \leq V(0)<\infty$, implying $V$ is bounded and thus $\{\mathbf{s}, \tilde{\mathbf{a}}\} \in \mathcal{L}_{\infty}$. Integrating 29 from $t=0$ to $t=T \geq 0$ gives

$$
\begin{gathered}
\int_{0}^{T} V \mathrm{~d} t \leq-\int_{0}^{T} \mathbf{s}^{\top} \mathbf{K} \mathbf{s} \mathrm{d} t \\
V(T)-V(0) \leq-\lambda_{\min }(\mathbf{K})\|\mathbf{s}\|_{2 T}^{2},
\end{gathered}
$$


which is rearranged to yield

$$
\lambda_{\min }(\mathbf{K})\|\mathbf{s}\|_{2 T}^{2} \leq V(0)-V(T)<\infty
$$

Since $\lambda_{\min }(\mathbf{K})>0$, it is concluded that $\mathbf{s} \in \mathcal{L}_{2} \cap \mathcal{L}_{\infty}$.

The second feedback controller considered in this thesis is an SPR controller, where the control law

$$
\overline{\mathbf{u}}=-\mathbf{y}_{c}
$$

is defined based on the output of the SPR transfer matrix $\mathbf{G}_{c}(s)=\mathbf{C}_{c}\left(s \mathbf{1}-\mathbf{A}_{c}\right)^{-1} \mathbf{B}_{c}$ with state-space realization

$$
\dot{\mathbf{x}}_{c}=\mathbf{A}_{c} \mathbf{x}_{c}+\mathbf{B}_{c} \mathbf{s}, \quad \mathbf{y}_{c}=\mathbf{C}_{c} \mathbf{x}_{c}
$$

The SPR property of the transfer matrix $\mathbf{G}_{c}(s)$ ensures that there exists a matrix $\mathbf{P}=\mathbf{P}^{\top}>0$ satisfying

$$
\mathbf{P A}_{c}+\mathbf{A}_{c}^{\top} \mathbf{P}=-\mathbf{Q}, \quad \mathbf{P B}_{c}=\mathbf{C}_{c}^{\top}
$$

where $\mathbf{Q}=\mathbf{Q}^{\top}>0\lceil 14$.

Theorem 4. Consider the tower crane system described by the equations of motion (6) and (5). The control law defined by (15) and (30) ensures that $\mathbf{s} \in \mathcal{L}_{2} \cap \mathcal{L}_{\infty}$.

Proof. Consider the function $\bar{V}=\frac{1}{2} \mathbf{x}_{c}^{\top} \mathbf{P} \mathbf{x}_{c}+V$, where $V$ is defined in (21) and $\mathbf{P}=\mathbf{P}^{\top}>0$, which ensures that $\bar{V}$ is nonnegative. Taking the time derivative of $\bar{V}$ 
and substituting in the dynamics of the SPR controller in 31) results in

$$
\dot{\bar{V}}=\frac{1}{2} \mathbf{x}_{c}^{\top}\left(\mathbf{P} \mathbf{A}_{c}+\mathbf{A}_{c}^{\top} \mathbf{P}\right) \mathbf{x}_{c}+\mathbf{x}_{c}^{\top} \mathbf{P} \mathbf{B}_{c} \mathbf{s}+\dot{V}
$$

Substituting the inequality for $\dot{V}$ from (24) and the properties of the SPR controller from (31) into this expression yields

$$
\begin{aligned}
\dot{\bar{V}} & \leq \frac{1}{2} \mathbf{x}_{c}^{\top}\left(\mathbf{P} \mathbf{A}_{c}+\mathbf{A}_{c}^{\top} \mathbf{P}\right) \mathbf{x}_{c}+\mathbf{x}_{c}^{\top} \mathbf{P} \mathbf{B}_{c} \mathbf{s}+\mathbf{s}^{\top} \overline{\mathbf{u}}-\mathbf{s}^{\top} \overline{\mathbf{M}} \lambda \mathbf{S} \\
& \leq-\frac{1}{2} \mathbf{x}_{c}^{\top} \mathbf{Q} \mathbf{x}_{c}-\mathbf{x}_{c}^{\top} \mathbf{C}_{c}^{\top} \mathbf{S}-\mathbf{s}^{\top} \mathbf{C}_{c} \mathbf{x}_{c}-\epsilon \mathbf{S}^{\top} \mathbf{S} \\
& \leq-\frac{1}{2} \lambda_{\min }(\mathbf{Q}) \mathbf{x}_{c}^{\top} \mathbf{x}_{c}-\epsilon \mathbf{S}^{\top} \mathbf{s} .
\end{aligned}
$$

Since $\epsilon>0$ and $\lambda_{\min }(\mathbf{Q})>0$ from the positive definiteness of $\mathbf{Q}$, it is known that $\dot{\bar{V}} \leq 0$. This implies that $0 \leq \bar{V}(T) \leq \bar{V}(0)<\infty$, ensuring $\bar{V}$ is bounded, and thus $\left\{\mathbf{s}, \tilde{\mathbf{a}}, \mathbf{x}_{c}\right\} \in \mathcal{L}_{\infty}$. Integrating $\dot{\bar{V}}$ from $t=0$ to $t=T \geq 0$ gives

$$
\begin{aligned}
\int_{0}^{T} \dot{\bar{V}} \mathrm{~d} t & \leq-\int_{0}^{T}\left(\frac{1}{2} \lambda_{\min }(\mathbf{Q}) \mathbf{x}_{c}^{\top} \mathbf{x}_{c}+\epsilon \mathbf{s}^{\top} \mathbf{s}\right) \mathrm{d} t \\
\bar{V}(T)-\bar{V}(0) & \leq-\frac{1}{2} \lambda_{\min }(\mathbf{Q}) \int_{0}^{T} \mathbf{x}_{c}^{\top} \mathbf{x}_{c} \mathrm{~d} t-\epsilon \int_{0}^{T} \mathbf{s}^{\top} \mathbf{s} \mathrm{d} t \\
& \leq-\frac{1}{2} \lambda_{\min }(\mathbf{Q})\left\|\mathbf{x}_{c}\right\|_{2 T}^{2}-\epsilon\|\mathbf{s}\|_{2 T}^{2} .
\end{aligned}
$$

Rearranging this expression results in

$$
\frac{1}{2} \lambda_{\min }(\mathbf{Q})\left\|\mathbf{x}_{c}\right\|_{2 T}^{2}+\epsilon|| \mathbf{s} \|_{2 T}^{2} \leq \bar{V}(0)-\bar{V}(T)<\infty
$$


and leads to the conclusion that $\left\{\mathbf{s}, \mathbf{x}_{c}\right\} \in \mathcal{L}_{2} \cap \mathcal{L}_{\infty}$.

Theorems 3 and 4 demonstrate that $\left\{\mathbf{s}, \mathbf{x}_{c}\right\} \in \mathcal{L}_{2} \cap \mathcal{L}_{\infty}$ when either the constant gain controller in $(28)$ or SPR feedback controller in $(30)$ is used. The following theorem makes use of this result to prove that the tracking errors are either bounded or asymptotically converge to zero.

Theorem 5. If $\mathbf{s} \in \mathcal{L}_{2} \cap \mathcal{L}_{\infty}$, then $\tilde{q}_{a, 1}, \tilde{q}_{a, 3} \in \mathcal{L}_{\infty}$ and $\tilde{q}_{a, 2} \rightarrow 0$ as $t \rightarrow \infty$.

Proof. It is assumed that the hoist cable sway angles are bounded within $\{\theta, \psi\} \in$ $\left(-\frac{\pi}{2}, \frac{\pi}{2}\right)$, ensuring $\mathbf{q}_{u} \in \mathcal{L}_{\infty}$. Rearranging and expanding (7) gives

$$
\begin{aligned}
& \dot{\tilde{q}}_{a, 1}=-\lambda_{1} \tilde{q}_{a, 1}-\alpha_{1} q_{u, 1}+s_{1}, \\
& \dot{\tilde{q}}_{a, 2}=-\lambda_{2} \tilde{q}_{a, 2}+s_{2}, \\
& \dot{\tilde{q}}_{a, 3}=-\lambda_{3} \tilde{q}_{a, 3}-\alpha_{2} q_{u, 2}+s_{3} .
\end{aligned}
$$

The terms $\mathbf{s}$ and $\mathbf{q}_{u}$ are both bounded (i.e., $\left\{\mathbf{s}, \mathbf{q}_{u}\right\} \in \mathcal{L}_{\infty}$ ). The ordinary differential equations in $(32)$ and $(34)$ can therefore be interpreted as linear time-invariant asymptotically systems whose inputs are in $\mathcal{L}_{\infty}$, and thus, $\left\{\tilde{q}_{a, 1}, \tilde{q}_{a, 3}, \dot{\tilde{q}}_{a, 1}, \dot{\tilde{q}}_{a, 3}\right\} \in \mathcal{L}_{\infty}$ [14, p. 270].

Because $q_{2} \in \mathcal{L}_{2}$ and $(33)$ is an asymptotically stable LTI system with an $\mathcal{L}_{2}$ input, $\tilde{q}_{a, 2} \in \mathcal{L}_{2} \cap \mathcal{L}_{\infty}, \dot{\tilde{q}}_{a, 2} \in \mathcal{L}_{2}$, and $\tilde{q}_{a, 2} \rightarrow 0$ as $t \rightarrow \infty$ 14, p. 269]

The result of Theorem 5 is the crux of the stability analysis, showing that the payload position tracking error asymptotically converges to zero in the $\ell$ coordinate 
and remains bounded in the $x$ and $\gamma$ coordinates. Note that if $\mathbf{q}_{u} \rightarrow \mathbf{0}$ as $t \rightarrow \infty$, then the tracking errors in all directions asymptotically converge to zero.

\subsection{Selection of Bounding Parameter}

As noted in the formulation of the adaptive control law in Section $4.2, \boldsymbol{\delta}$ must satisfy

the matrix inequality $\dot{\overline{\mathbf{M}}}(\mathbf{q}, \dot{\mathbf{q}})-\left(\dot{\mathbf{q}}^{\top} \boldsymbol{\delta}\right) \mathbf{1}<0$. A challenge in computing the values of $\boldsymbol{\delta}$ that satisfy this inequality comes from the fact that $\dot{\overline{\mathbf{M}}}(\mathbf{q}, \dot{\mathbf{q}})$ varies throughout the workspace (i.e., the range of possible values of $\mathbf{q}$ ) of the tower crane. The following lemma provides insight that is used to develop a novel method with which to select $\delta$.

Lemma 1. If $\boldsymbol{\delta} \in \mathbb{R}^{5}$ satisfies

$$
\lambda_{\max }\left(\frac{\partial \overline{\mathbf{M}}}{\partial q_{i}}\right)<\delta_{i}, i=1, \ldots, 5
$$

where $q_{i}$ and $\delta_{i}$ are the $i^{\text {th }}$ entries of $\mathbf{q}$ and $\boldsymbol{\delta}$, respectively, then $\dot{\overline{\mathbf{M}}}(\mathbf{q}, \dot{\mathbf{q}})-\left(\dot{\mathbf{q}}^{\top} \boldsymbol{\delta}\right) \mathbf{1}<0$.

Proof. The time derivative of $\overline{\mathbf{M}}(\mathbf{q})$ can be written as

$$
\dot{\overline{\mathbf{M}}}=\frac{\partial \overline{\mathbf{M}}}{\partial x} \dot{x}+\frac{\partial \overline{\mathbf{M}}}{\partial \ell} \dot{\ell}+\frac{\partial \overline{\mathbf{M}}}{\partial \gamma} \dot{\gamma}+\frac{\partial \overline{\mathbf{M}}}{\partial \theta} \dot{\theta}+\frac{\partial \overline{\mathbf{M}}}{\partial \psi} \dot{\psi}
$$

where the partial derivatives of $\mathbf{M}$ are symmetric owing to the fact that $\mathbf{M}$ is symmetric. The inequalities in (35) imply that the maximum eigenvalue of each partial derivative is strictly less than its corresponding entry of $\boldsymbol{\delta}$. This can be rewritten as 
the set of matrix inequalities

$$
\begin{array}{r}
\frac{\partial \overline{\mathbf{M}}}{\partial x}-\delta_{x} \mathbf{1}<0, \frac{\partial \overline{\mathbf{M}}}{\partial \ell}-\delta_{\ell} \mathbf{1}<0, \frac{\partial \overline{\mathbf{M}}}{\partial \gamma}-\delta_{\gamma} \mathbf{1}<0, \\
\frac{\partial \overline{\mathbf{M}}}{\partial \theta}-\delta_{\theta} \mathbf{1}<0, \frac{\partial \overline{\mathbf{M}}}{\partial \psi}-\delta_{\psi} \mathbf{1}<0
\end{array}
$$

Multiplying each inequality in (37) by its respective coordinate of $\dot{\mathbf{q}}$, summing them together, and substituting the result into 36 gives

$$
\dot{\overline{\mathbf{M}}}(\mathbf{q}, \dot{\mathbf{q}})-\left(\delta_{x} \dot{x}+\delta_{\ell} \dot{\ell}+\delta_{\gamma} \dot{\gamma}+\delta_{\theta} \dot{\theta}+\delta_{\psi} \dot{\psi}\right) \mathbf{1}<0
$$

which can be factored into $\dot{\overline{\mathbf{M}}}(\mathbf{q}, \dot{\mathbf{q}})-\left(\dot{\mathbf{q}}^{\top} \boldsymbol{\delta}\right) \mathbf{1}<0$.

In the work of [15], parameters similar to $\boldsymbol{\delta}$ that satisfy conditions similar to those given in Lemma 1 were solved for graphically in the case of a 2-DOF tower crane. This approach is not tractable in this thesis with a 5-DOF tower crane, as the partial derivatives are defined in five-dimensional space. Instead, a novel convex optimization approach is proposed to solve for $\boldsymbol{\delta}$ that minimizes conservatism in the inequalities of (35). The term $\boldsymbol{\delta}$ is included in the control law of $(12)$ only for the purpose of ensuring the tower crane system has an OSP input-output mapping, and does not directly contribute to the tracking of the desired payload trajectory. In other words, the selection of $\boldsymbol{\delta}$ represents a tradeoff between robustness and performance. Increased conservatism in the inequalities of (35) increases robustness in the passivity property of the system, while reduced conservatism in the inequalities of (35) potentially improves tracking performance.

Although the partial derivatives in (35) are not known exactly in practice, the 
model of the mass matrix $\overline{\mathbf{M}}(\mathbf{q})$ can be used to guide the choice of $\boldsymbol{\delta}$ in a manner that balances robustness and performance. The proposed method involves solving for $\boldsymbol{\delta}(\mathbf{q})$ as a surface parameterized in terms of $\mathbf{q}$. In this thesis, a quartic surface is defined as

$$
\boldsymbol{\delta}(\mathbf{q})=\Delta \overline{\mathbf{q}}(\mathbf{q})=\left[\begin{array}{c}
\Delta_{1} \\
\Delta_{2} \\
\Delta_{3} \\
\Delta_{4} \\
\Delta_{5}
\end{array}\right]\left[\begin{array}{c}
\mathbf{q}^{\circ 4} \\
\mathbf{q}^{\circ 3} \\
\mathbf{q}^{\circ 2} \\
\mathbf{q} \\
1
\end{array}\right],
$$

where $\mathbf{q}^{\circ n}$ denotes that each element of the matrix $\mathbf{q}$ is raised to the power of $n$ and $\boldsymbol{\Delta}_{i} \in \mathbb{R}^{1 \times 21}, i=1, \ldots, 5$ contain the surface coefficients. The surface coefficients are solved by first defining a grid of points within the tower crane's workspace, where numerical ranges for the generalized coordinates $\mathbf{q}$ are given in Table 1. Each row of $\Delta$ is then calculated by solving the constrained least squares problem

$$
\boldsymbol{\Delta}_{i}^{\star}=\arg \min _{\mathbf{A} \boldsymbol{\Delta}_{i}^{\top} \geq \mathbf{b}}\left\|\mathbf{A} \boldsymbol{\Delta}_{i}^{\top}-\mathbf{b}\right\|^{2}
$$

where

$$
\mathbf{A}=\left[\begin{array}{ccccc}
\mathbf{q}_{1}^{\circ 4} & \mathbf{q}_{1}^{\circ 3} & \mathbf{q}_{1}^{\circ 2} & \mathbf{q}_{1} & 1 \\
\vdots & \vdots & \vdots & \vdots & \vdots \\
\mathbf{q}_{n}^{\circ 4} & \mathbf{q}_{n}^{\circ 3} & \mathbf{q}_{n}^{\circ 2} & \mathbf{q}_{n} & 1
\end{array}\right]
$$

contains the $n$ grid points and $\mathbf{b}^{\top}=\left[\begin{array}{lll}\lambda_{i, 1}+\epsilon_{i, 1} & \cdots & \lambda_{i, n}+\epsilon_{i, n}\end{array}\right]$ contains the corresponding maximum eigenvalues of $\dot{\overline{\mathbf{M}}}$ evaluated at the grid points with robustness margin $\epsilon \geq 0$ added. Using a fine enough grid and allowing for a large enough margin to compensate for inexact knowledge of the system parameters ensures that (13) is satisfied robustly. 


\section{Numerical Results}

Although Theorem 5 shows that all coordinate errors are $\in \mathcal{L}_{\infty}$ and notes that if $\lim _{t \rightarrow \infty} \mathbf{q}_{u}=\mathbf{0} \Longrightarrow \lim _{t \rightarrow \infty} \tilde{\mathbf{q}}_{a}=\mathbf{0}$, it provides no basis for ensuring that $\lim _{t \rightarrow \infty} \mathbf{q}_{u}=\mathbf{0}$ is satisfied. Supporting evidence via a linearization of the error dynamics is provided to support this satisfaction.

Combining and rearranging (18) and (10) gives

$$
\overline{\mathbf{M}}\left(\ddot{\mathbf{q}}_{a}+2 \boldsymbol{\lambda} \dot{\mathbf{q}}_{a}+\boldsymbol{\lambda}^{\top} \boldsymbol{\lambda} \tilde{\mathbf{q}}_{a}+\boldsymbol{\alpha} \dot{\mathbf{q}}_{u}+\boldsymbol{\lambda} \boldsymbol{\alpha} \mathbf{q}_{u}\right)+\overline{\mathbf{u}}+\tilde{\mathbf{C}}_{a} \dot{\mathbf{q}}_{a}+\tilde{\mathbf{C}}_{u} \dot{\mathbf{q}}_{u}+\tilde{\mathbf{G}}-\frac{1}{2}\left(\dot{\mathbf{q}}^{\top} \boldsymbol{\delta}\right) \mathbf{s}=\mathbf{0}
$$

Linearizing about $\dot{\mathbf{q}}=\mathbf{0}$, substituting in the constant-gain controller (28), and simplifying gives

$$
\begin{gathered}
\overline{\mathbf{M}}\left(\ddot{\mathbf{q}}_{a}+2 \boldsymbol{\lambda} \dot{\mathbf{q}}_{a}+\boldsymbol{\lambda}^{\top} \boldsymbol{\lambda} \tilde{\mathbf{q}}_{a}+\boldsymbol{\alpha} \dot{\mathbf{q}}_{u}+\boldsymbol{\lambda} \boldsymbol{\alpha} \mathbf{q}_{u}\right)-\mathbf{K s}=\mathbf{0} \\
\overline{\mathbf{M}} \ddot{\mathbf{q}}_{a}+\overline{\mathbf{M}} \boldsymbol{\alpha} \dot{\mathbf{q}}_{u}+(2 \overline{\mathbf{M}} \boldsymbol{\lambda}-\mathbf{K}) \dot{\mathbf{q}}_{a}+\left(\overline{\mathbf{M}} \boldsymbol{\lambda}^{\top} \boldsymbol{\lambda}-\mathbf{K} \boldsymbol{\lambda}\right) \tilde{\mathbf{q}}_{a}+(\overline{\mathbf{M}} \boldsymbol{\lambda} \boldsymbol{\alpha}-\mathbf{K} \boldsymbol{\alpha}) \mathbf{q}_{u}=\mathbf{0}
\end{gathered}
$$

Combining this with the reformulated equations of motion for the unactuated coordinates (5) and linearizing about a desired point $\overline{\mathbf{q}}^{\top}=\left[\begin{array}{lllll}x_{d} & \ell_{d} & \gamma_{d} & 0 & 0\end{array}\right], \overline{\dot{\mathbf{q}}}=\overline{\ddot{\mathbf{q}}}=\mathbf{0}$ gives the linearized closed-loop equations of motion. These can be grouped into the form

$$
\mathbf{M}_{l i n} \ddot{\mathbf{q}}+\mathbf{C}_{l i n} \dot{\mathbf{q}}+\mathbf{K}_{l i n} \tilde{\mathbf{q}}=\mathbf{0}
$$


where

$$
\begin{gathered}
\mathbf{M}_{l i n}=\left[\begin{array}{cc}
\overline{\mathbf{M}}(\overline{\mathbf{q}}) & \mathbf{0} \\
\mathbf{M}_{u a}(\overline{\mathbf{q}}) & \mathbf{M}_{u u}(\overline{\mathbf{q}})
\end{array}\right], \\
\mathbf{C}_{l i n}=\left[\begin{array}{cc}
2 \overline{\mathbf{M}}(\overline{\mathbf{q}}) \boldsymbol{\lambda}-\mathbf{K} & \overline{\mathbf{M}}(\overline{\mathbf{q}}) \boldsymbol{\alpha} \\
-\mathbf{C}_{u a}(\overline{\mathbf{q}}, \mathbf{0}) & \mathbf{D}_{u}-\mathbf{C}_{u u}(\overline{\mathbf{q}}, \mathbf{0})
\end{array}\right], \\
\mathbf{K}_{l i n}=\left[\begin{array}{cc}
\overline{\mathbf{M}}(\overline{\mathbf{q}}) \boldsymbol{\lambda}^{\top} \boldsymbol{\lambda}-\mathbf{K} \boldsymbol{\lambda} & \overline{\mathbf{M}}(\overline{\mathbf{q}}) \boldsymbol{\lambda} \boldsymbol{\alpha}-\mathbf{K} \boldsymbol{\alpha} \\
\mathbf{0} & \left.\frac{\partial \mathbf{G}_{u}}{\partial \mathbf{q}_{u}}\right|_{\mathbf{q}=\overline{\mathbf{q}}}
\end{array}\right] .
\end{gathered}
$$

Defining the state variable $\mathbf{x}^{\top}=\left[\begin{array}{ll}\mathbf{q}^{\top} & \dot{\mathbf{q}}^{\top}\end{array}\right]$ forms the closed-loop linear system

$$
\dot{\mathbf{x}}=\mathbf{A}_{C L} \mathbf{x}=\left[\begin{array}{cc}
\mathbf{0} & \mathbf{1} \\
-\mathbf{M}_{l i n}^{-1} \mathbf{K}_{l i n} & -\mathbf{M}_{l i n}^{-1} \mathbf{C}_{l i n}
\end{array}\right] \mathbf{x}
$$

Because the behavior of the crane is invariant to the value of $\gamma$, this linearization is performed for varying values of $x$ and $\ell$ using the gains and system parameters specified in section 6 and the maximum real part of the eigenvalues of $\mathbf{A}_{C L}$ recorded. The results are displayed in figure 4.

Because the maximum real eigenvalue part over the workspace is less than 0 , we conclude that the linearized system is stable. Although this is not a rigorous proof of stability, it supports the other evidence that in a practical sense, the sway angle will eventually become zero.

\section{Experimental Results}

In this section a comparison to the adaptive output feedback control method presented in [8] is provided. To the authors' knowledge this is the only other proposed 


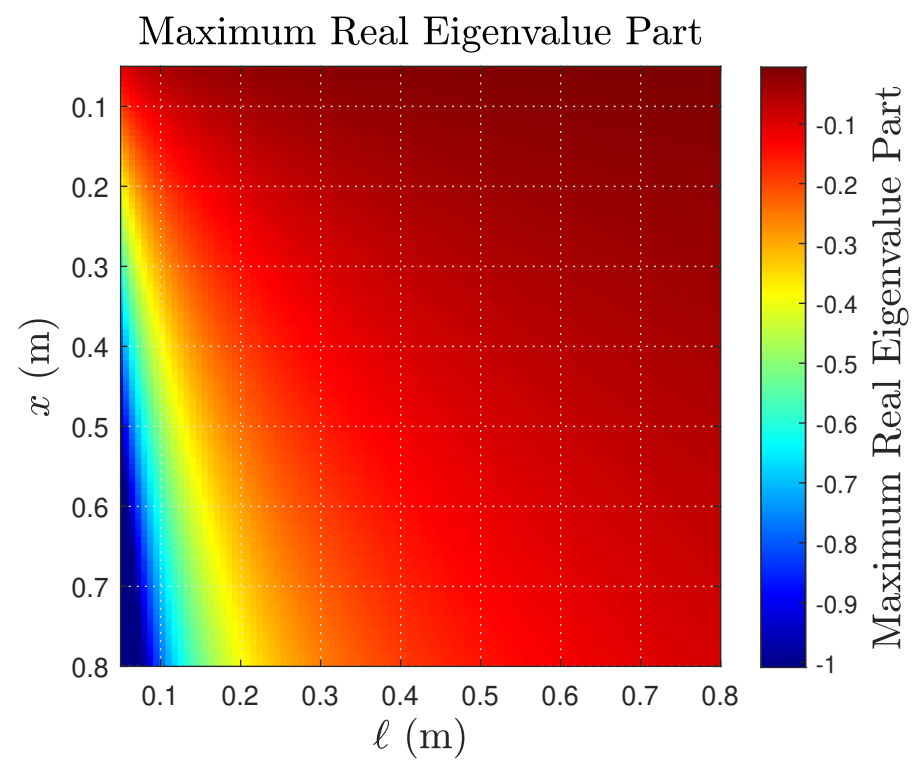

Figure 4: Maximum real eigenvalue part of the linearized closed-loop system

control law to perform 5-DOF tower crane control using similar control methods.

The adaptive output feedback control method from [8] uses a control law of the form

$$
\mathbf{u}=-\boldsymbol{\lambda}_{p} \tilde{\mathbf{q}}_{a}+\boldsymbol{\lambda}_{v, 1}\left(\mathbf{q}_{a}-\mathbf{q}_{v}\right)-\left[\begin{array}{c}
0 \\
y(\ell)+\hat{m}_{c} g \\
0
\end{array}\right]
$$

where $\boldsymbol{\lambda}_{p}=\operatorname{diag}\left\{\lambda_{p, 1}, \lambda_{p, 2}, \lambda_{p, 3}\right\}$ are positive control gains,

$$
\begin{gathered}
y(\ell)=-k_{\ell} \frac{\frac{\arctan \left(\ell_{M}-\ell\right)}{1+\left(\ell-\ell_{m}\right)^{2}}-\frac{\arctan \left(\ell-\ell_{m}\right)}{1+\left(\ell_{M}-\ell\right)^{2}}}{\arctan ^{2}\left(\ell-\ell_{m}\right) \arctan ^{2}\left(\ell_{M}-\ell\right)} \tilde{\ell}^{2}+\frac{2 k_{\ell} \tilde{\ell}}{\arctan \left(\ell-\ell_{m}\right) \arctan \left(\ell_{M}-\ell\right)}, \\
\hat{m}_{c}=\frac{g}{k_{\mathrm{m} 1}}(\ell-\ell(0))+k_{\mathrm{m} 2} \frac{\left(\int_{0}^{t} \tilde{\ell}(\tau) \mathrm{d} \tau\right)^{2}}{1+\left(\int_{0}^{t} \tilde{\ell}(\tau) \mathrm{d} \tau\right)^{2}},
\end{gathered}
$$


Table 1: Workspace of the tower crane used in experiments.

\begin{tabular}{c|c|c|c}
\hline Coordinate & Symbol & Range & Units \\
\hline Cart Position & $x$ & $(0,0.75]$ & $\mathrm{m}$ \\
Cable Length & $\ell$ & $(0,1.202]$ & $\mathrm{m}$ \\
Jib Angle & $\gamma$ & $(-\pi, \pi]$ & $\mathrm{rad}$ \\
Radial Sway Angle & $\theta$ & $\left(-\frac{\pi}{2}, \frac{\pi}{2}\right)$ & $\mathrm{rad}$ \\
Tangential Sway Angle & $\psi$ & $\left(-\frac{\pi}{2}, \frac{\pi}{2}\right)$ & $\mathrm{rad}$ \\
\hline
\end{tabular}

Table 2: Numerical parameters of the tower crane used in experiments.

\begin{tabular}{c|c|c|c}
\hline Parameter & Symbol & Value & Units \\
\hline Cart Mass & $m_{1}$ & 0.2238 & $\mathrm{~kg}$ \\
Payload Mass & $m_{2}$ & 0.147 & $\mathrm{~kg}$ \\
Spool Radius & $r_{s}$ & 0.0148 & $\mathrm{~m}$ \\
Spool Inertia & $J_{s}$ & $3.64 \cdot 10^{-6}$ & $\mathrm{~kg} \cdot \mathrm{m}^{2}$ \\
Jib Inertia & $J_{j}$ & 0.8872 & $\mathrm{~kg} \cdot \mathrm{m}^{2}$ \\
Gravity & $g$ & 9.81 & $\mathrm{~m} \cdot \mathrm{s}^{-2}$ \\
\hline
\end{tabular}

and $k_{\ell}, k_{\mathrm{m} 1}, k_{\mathrm{m} 2} \in \mathbb{R}_{\geq 0} . \mathbf{q}_{v}$ is defined by a virtual mass-spring system

$$
\mathbf{m}_{v} \ddot{\mathbf{q}}_{v}=-\boldsymbol{\lambda}_{v_{1}}\left(\mathbf{q}_{v}-\mathbf{q}_{a}\right)+\boldsymbol{\lambda}_{v, 2}\left(\mathbf{q}_{d}-\mathbf{q}_{v}\right)-\boldsymbol{\Phi} \dot{\mathbf{q}}_{v}
$$

where $\boldsymbol{\lambda}_{v, 1}=\operatorname{diag}\left\{\lambda_{v 1,1}, \lambda_{v 1,2}, \lambda_{v 1,3}\right\}>0, \boldsymbol{\lambda}_{v, 2}=\operatorname{diag}\left\{\lambda_{v 2,1}, \lambda_{v 2,2}, \lambda_{v 2,3}\right\}>0$ are spring stiffnesses, $\boldsymbol{\Phi}=\operatorname{diag}\left\{\Phi_{1}, \Phi_{2}, \Phi_{3}\right\}>0$ are spring damping coefficients, and $\mathbf{x}_{v}=$ $\operatorname{diag}\left\{m_{1}, m_{2}, m_{3}\right\}>0$ are virtual spring masses. Note that the adaptive control law in [8] does not include any terms explicitly accounting for the payload sway $\mathbf{q}_{u}$.

The tower crane used for experiments is the Quanser "3 DOF" lab tower crane 21. This crane has the physical parameters and workspace as described in tables 2 and 1 respectively. The tower crane is equipped with encoders to measure trolley position, hoist cable winch rotation (hoist cable length), jib angle, and both sway angles. Time derivatives are not measured directly but calculated through filtered derivatives of position. 


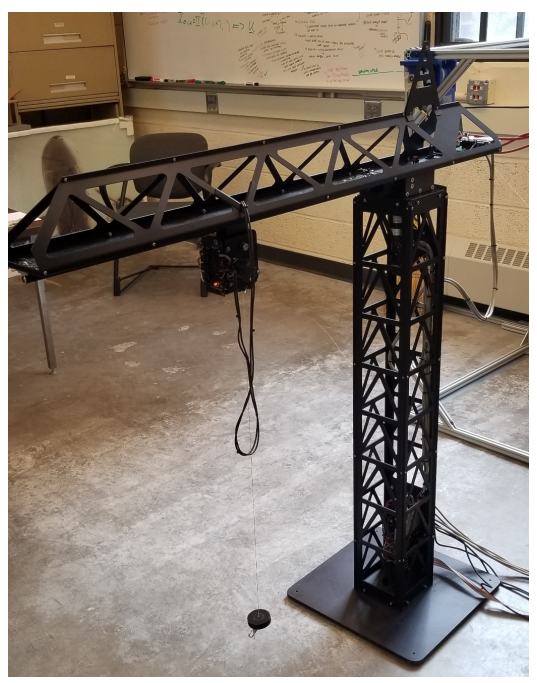

Figure 5: Picture of the Quanser tower crane used in section 6.

Table 3: Initial and final positions of the desired payload trajectories used in experiments as a function of time.

\begin{tabular}{|c|c|c|}
\hline Time (s) & $\boldsymbol{\rho}_{i}^{\top}$ & $\boldsymbol{\rho}_{f}^{\mathbf{T}}$ \\
\hline $0<t<4$ & {$\left[\begin{array}{lll}0.43 & 0.8636 & 0\end{array}\right]$} & {$\left[\begin{array}{lll}0 & 0.4 & 0.5\end{array}\right]$} \\
\hline $8 \leq t \leq 12$ & {$\left[\begin{array}{lll}0 & 0.4 & 0.5\end{array}\right]$} & {$\left[\begin{array}{lll}-0.3 & 0.8 & 0.3\end{array}\right]$} \\
\hline $16 \leq t \leq 20$ & {$\left[\begin{array}{lll}-0.3 & 0.8 & 0.3\end{array}\right]$} & {$\left[\begin{array}{lll}0 & 0.3 & 0.38\end{array}\right]$} \\
\hline $24 \leq t \leq 28$ & {$\left[\begin{array}{lll}0 & 0.3 & 0.38\end{array}\right]$} & $\begin{array}{lll}0.25 & 0.3 & 0\end{array}$ \\
\hline $32 \leq t \leq 36$ & {$\left[\begin{array}{lll}0.25 & 0.3 & 0\end{array}\right]$} & {$\left[\begin{array}{lll}0 & 0.8 & -0.6\end{array}\right]$} \\
\hline $40 \leq t \leq 44$ & {$\left[\begin{array}{lll}-0.3 & 0.8 & 0.3\end{array}\right]$} & {$\left[\begin{array}{lll}0.43 & 0.8636 & 0\end{array}\right]$} \\
\hline $44 \leq t \leq 56$ & {$\left[\begin{array}{lll}0.43 & 0.8636 & 0\end{array}\right]$} & {$\left[\begin{array}{lll}0.43 & 0.8636 & 0\end{array}\right]$} \\
\hline
\end{tabular}

Six four-second trajectories with four-second rest periods in between were performed encompassing a wide range of operating conditions. The last rest period is instead twelve seconds to allow the cable sway to be neutralized. Results are given in the frame $\mathcal{F}_{c}$. The proposed control law is implemented with both an SPR controller (30) and constant gain controller (28), and gains $\boldsymbol{\lambda}=\operatorname{diag}\left\{25 \mathrm{~s}^{-1}, 50 \mathrm{~s}^{-1}, 50 \mathrm{~m} / \mathrm{s}\right\}$, 
$\mathbf{K}=\operatorname{diag}\{20 \mathrm{~N} \cdot \mathrm{s} / \mathrm{m}, 20 \mathrm{~N} \cdot \mathrm{s} / \mathrm{m}, 100 \mathrm{~N} \cdot \mathrm{s}\}, \boldsymbol{\Gamma}=\mathbf{1}$

$$
\boldsymbol{\alpha}=\left[\begin{array}{cc}
4 & 0 \\
0 & 0 \\
0 & 10
\end{array}\right] \mathrm{m} / \mathrm{s}
$$

The transfer function of the SPR controller is

$$
\mathbf{G}_{c}(s)=\mathbf{K} \operatorname{diag}\left\{\frac{a}{s+a}, \frac{a}{s+a}, \frac{a}{s+a}\right\},
$$

with $a=10 \mathrm{rad} / \mathrm{s}$. The control gains for the adaptive controller from [8] are $\boldsymbol{\lambda}_{p}=$ $\operatorname{diag}\{860 \mathrm{~N} / \mathrm{m}, 4400 \mathrm{~N} / \mathrm{m}, 3500 \mathrm{~N} \cdot \mathrm{m}\}, \boldsymbol{\lambda}_{v, 1}=\operatorname{diag}\{16 \mathrm{~N} / \mathrm{m}, 20 \mathrm{~N} / \mathrm{m}, 1200 \mathrm{~N} \cdot \mathrm{m}\}$, $\boldsymbol{\lambda}_{v, 2}=\operatorname{diag}\{5 \mathrm{~N} / \mathrm{m}, 0.05 \mathrm{~N} / \mathrm{m}, 5 \mathrm{~N} \cdot \mathrm{m}\}, \mathbf{m}_{v}=\operatorname{diag}\left\{1 \mathrm{~kg}, 1 \mathrm{~kg}, 1 \mathrm{~kg} \cdot \mathrm{m}^{2}\right\}$, $\boldsymbol{\Phi}=\operatorname{diag}\{1 \mathrm{~N} \cdot \mathrm{s} / \mathrm{m}, 3 \mathrm{~N} \cdot \mathrm{s} / \mathrm{m}, 1 \mathrm{~N} \cdot \mathrm{m} \cdot \mathrm{s}\}, k_{\ell}=0.01 \mathrm{~N} / \mathrm{m}, k_{m, 1}=10 \mathrm{~kg}^{-1} \mathrm{~s}^{-4}$, and $k_{m, 2}=2 \mathrm{~kg} / \mathrm{m}$

All adaptive parameters for both controllers are initialized as containing entirely 0.

Results are shown in Figures 6, 7, 8, and 9. The results from the constant and SPR controllers are listed as K and SPR respectively. The results from the adaptive controller from [8 are listed as $\mathrm{Wu} 2021$. In results with a trajectory, this is listed as $\rho_{d}$. Both the proposed controller and the adaptive controller from [8] adequately track the trajectory, however the adaptive controller from [8] is unable to damp the payload sway either during or at the end of the trajectory. Figure 9 shows that the feedback control of the proposed controller is minimal compared to the adaptive control, showing that the dynamics are well-captured by the adaptation system. There is minimal difference between the two feedback controllers, as feedback is 

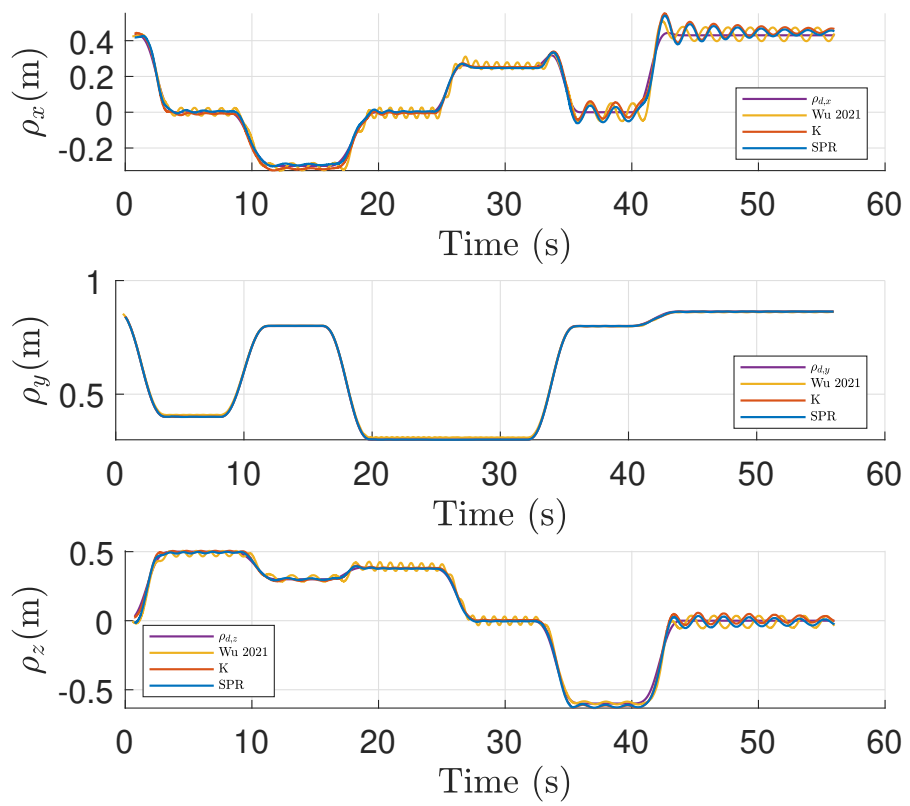

Figure 6: Payload positions over time with both controllers.
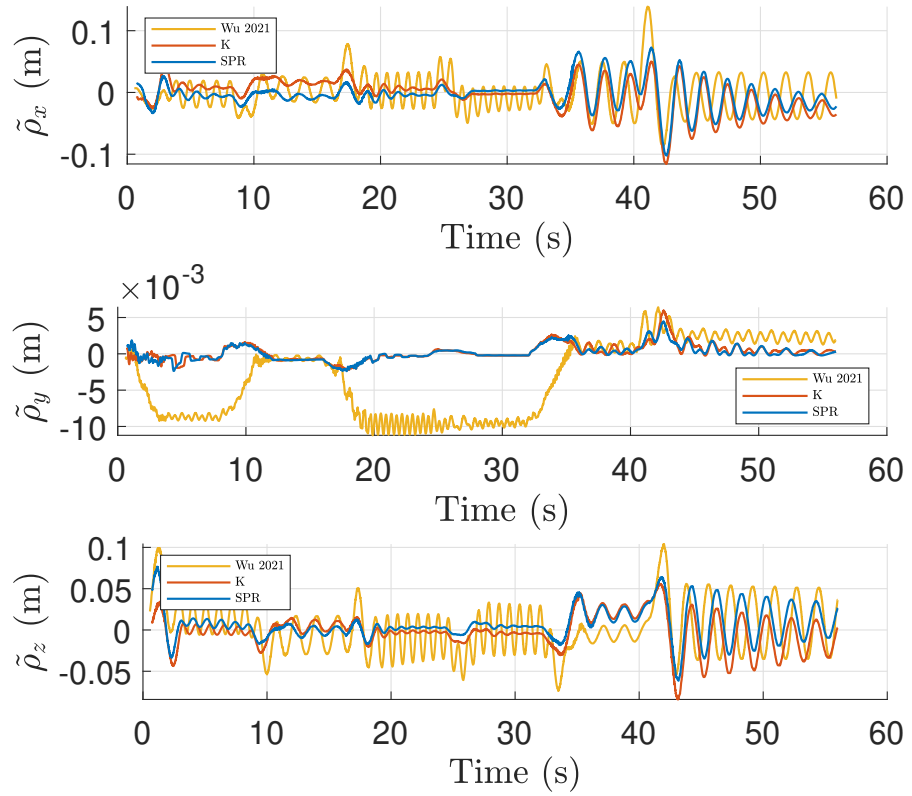

Figure 7: Payload position errors over time with both controllers. 

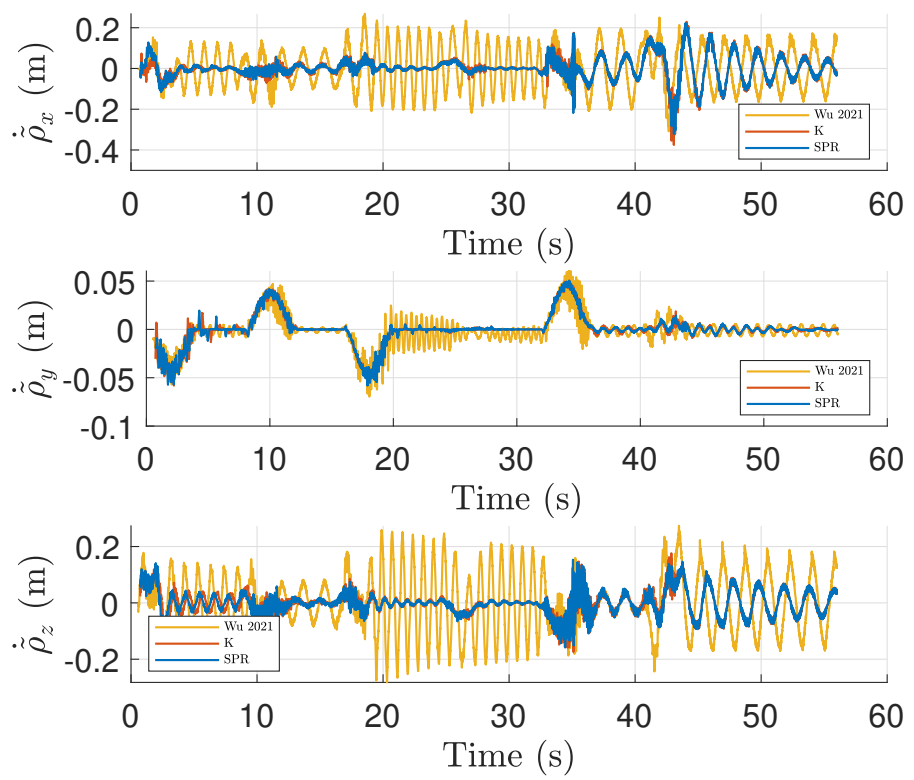

Figure 8: Payload velocity errors over time with both controllers.
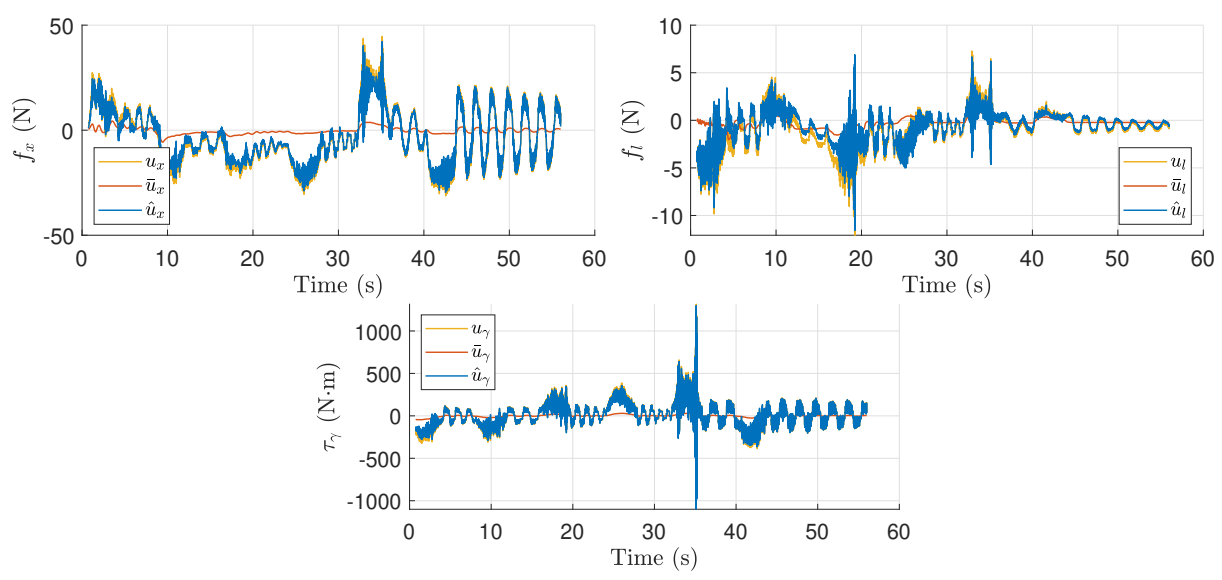

Figure 9: SPR controller forces over time. 

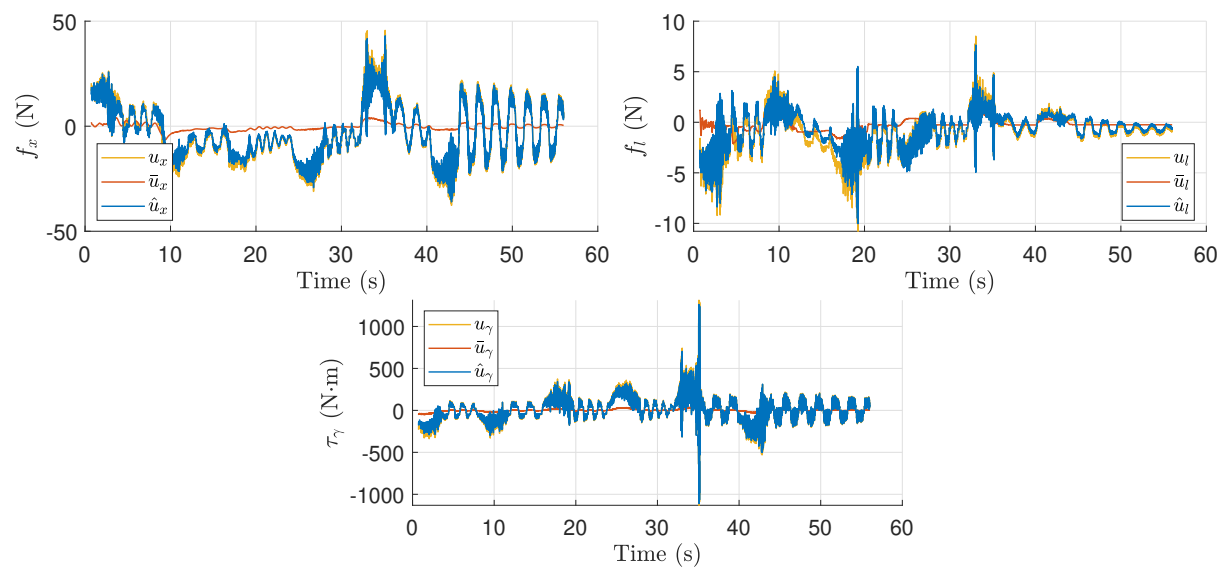

Figure 10: Constant gain controller forces over time.

minimized throughout the trajectory.

There is a minor amount of steady-state error by the two proposed controller variants, possibly as a result of unmodeled static friction.

\section{Conclusion}

A novel passivity-based adaptive controller for payload trajectory tracking of a 5DOF tower crane was developed and validated in this thesis. A key contribution of this work is a procedure that optimally bounds the derivative of the system mass matrix, which ensures that the system is OSP and closed-loop input-output stability can be proven using the Passivity Theorem. Experimental results show that the controller improves on the state of the art in tracking trajectories in a wide range of operating conditions and in damping payload sway. The ability of the controller to damp payload sway is further supported by a numerical linearization of the closedloop system showing negative eigenvalues. 
Extensions of this work could include optimal feedback controller design, gainscheduled sliding surface weights, and actuator saturation.

\section{References}

[1] Y.-G. Sung and W. Singhose, "Robustness analysis of input shaping commands for two-mode flexible systems," IET Control Theory $\&$ Applications, vol. 3, no. 6, pp. 722-730, 2009.

[2] W. Singhose, L. Porter, M. Kenison, and E. Kriikku, "Effects of hoisting on the input shaping control of gantry cranes," Control Engineering Practice, vol. 8, no. 10, pp. 1159-1165, 2000.

[3] D. Blackburn, J. Lawrence, J. Danielson, W. Singhose, T. Kamoi, and A. Taura, "Radial-motion assisted command shapers for nonlinear tower crane rotational slewing," Control Engineering Practice, vol. 18, no. 5, pp. 523-531, 2010.

[4] M. Zhang, Y. Zhang, and X. Cheng, "Model-free adaptive integral sliding mode control for 4-DOF tower crane systems," in IEEE/ASME International Conference on Advanced Intelligent Mechatronics (AIM), 2019, pp. 708-713.

[5] M. Böck and A. Kugi, "Real-time nonlinear model predictive path-following control of a laboratory tower crane," IEEE Transactions on Control Systems Technology, vol. 22, no. 4, pp. 1461-1473, 2014.

[6] K. Takagi and H. Nishimura, "Gain-scheduled control of a tower crane considering varying load-rope length," JSME International Journal Series C Mechani- 
cal Systems, Machine Elements and Manufacturing, vol. 42, no. 4, pp. 914-921, 1999.

[7] N. Sun, Y. Fang, H. Chen, B. Lu, and Y. Fu, "Slew/translation positioning and swing suppression for 4-DOF tower cranes with parametric uncertainties: Design and hardware experimentation," IEEE Transactions on Industrial Electronics, vol. 63, no. 10, pp. 6407-6418, 2016.

[8] Y. Wu, N. Sun, H. Chen, and Y. Fang, "Adaptive output feedback control for 5-dof varying-cable-length tower cranes with cargo mass estimation," IEEE Transactions on Industrial Informatics, vol. 17, no. 4, pp. 2453-2464, 2020.

[9] P.-Y. Shen and R. J. Caverly, "Noncolocated passivity-based control of a 2 DOF tower crane with a flexible hoist cable," in American Control Conference (ACC), 2020, pp. 5046-5051.

[10] R. Ortega and M. W. Spong, "Adaptive motion control of rigid robots: A tutorial," Automatica, vol. 25, no. 6, pp. 877-888, 1989.

[11] R. Ortega, A. Loría, P. J. Nicklasson, and H. Sira-Ramírez, Passivity-Based Control of Euler-Lagrange Systems: Mechanical, Electrical and Electromechanical Applications. London, UK: Springer-Verlag, 1998.

[12] C. J. Damaren, "Adaptive control of flexible manipulators carrying large uncertain payloads," Journal of Robotic Systems, vol. 13, no. 4, pp. 219-228, 1996. 
[13] R. J. Caverly and J. R. Forbes, "Dynamic modeling and noncollocated control of a flexible planar cable-driven manipulator," IEEE Transactions on Robotics, vol. 30, no. 6, pp. 1386-1397, 2014.

[14] B. Brogliato, R. Lozano, B. Maschke, and O. Egeland, Dissipative Systems Analysis and Control: Theory and Applications, ser. Communications and Control Engineering. Cham, Switzerland: Springer International Publishing AG, 2019.

[15] P.-Y. Shen, "Dynamic modeling and passivity-based control of a 2 DOF tower crane with a flexible hoist cable," Master's thesis, University of Minnesota, Minneapolis, MN, 2020.

[16] C. A. Desoer, Feedback systems: input-output properties, ser. Electrical science. New York: Academic Press, 1975.

[17] S. Boyd, L. El Ghaoui, E. Feron, and V. Balakrishnan, Linear matrix inequalities in system and control theory. Philadelphia, PA: SIAM, 1994.

[18] Q. H. Ngo and K.-S. Hong, "Adaptive sliding mode control of container cranes," IET Control Theory \& Applications, vol. 6, no. 5, pp. 662-668, 2012.

[19] L. A. Tuan, S.-C. Moon, W. G. Lee, and S.-G. Lee, "Adaptive sliding mode control of overhead cranes with varying cable length," Journal of Mechanical Science and Technology, vol. 27, no. 3, pp. 885-893, 2013.

[20] C. J. Damaren, "Passivity and noncollocation in the control of flexible multibody systems," Journal of Dynamic Systems, Measurement, and Control, vol. 122, no. 1, pp. 11-17, 2000. 
[21] Quanser, "3 dof crane user manual," 2013. [Online]. Available: https: //www.quanser.com/products/3-dof-crane/

\section{A Mass Matrix and Nonlinear Force Terms}

Details regarding the matrices that define the equations of motion of the tower crane are provided in this Appendix. The upper triangular entries of the mass matrix $\mathbf{M}(\mathbf{q})$ 
are given as

$$
\begin{aligned}
& M_{11}=m_{1}+m_{2}+2 \frac{J_{s}}{r_{s}^{2}}, M_{12}=m_{2} \cos (\psi) \sin (\theta), \\
& M_{13}=-m_{2}(\ell(\cos (\psi) \sin (2 \gamma) \sin (\theta) \\
& +\cos (2 \gamma) \sin \psi)+x \sin (2 \gamma)) \\
& M_{14}=-m_{2} \ell \cos (\theta) \cos (\psi), M_{15}=-m_{2} \ell \sin (\theta) \sin (\psi), \\
& M_{22}=m_{2}+\frac{J_{s}}{r_{s}^{2}} \\
& M_{23}=-m_{2} \ell \sin (2 \gamma)\left(\sin ^{2}(\theta) \cos ^{2}(\psi)+\sin ^{2}(\psi)\right) \\
& +x m_{2}(\sin (2 \gamma) \sin (\theta) \cos (\psi)-\cos (2 \gamma) \sin (\psi)), \\
& M_{24}=0, M_{25}=0 \text {, } \\
& M_{33}=J_{j}+x^{2}\left(\frac{2 J_{s}}{r_{s}^{2}}+m_{1}+m_{2}\right)+m_{2} \ell^{2}\left(\sin ^{2}(\theta) \cos ^{2}(\psi)\right. \\
& \left.+\sin ^{2}(\psi)\right)+2 m_{2} \ell x \sin (\theta) \cos (\psi), \\
& M_{34}=m_{2} \ell \cos (\theta) \cos (\psi)(\ell(\sin (2 \gamma) \sin (\theta) \cos (\psi) \\
& +\cos (2 \gamma) \sin (\psi))+x \sin (2 \gamma)) \\
& M_{35}=m_{2} \ell\left(\ell \left(\sin (\gamma) \cos (\gamma) \cos ^{2}(\theta) \sin (2 \psi)\right.\right. \\
& \left.+\sin ^{2}(\gamma) \sin (\theta)+\cos ^{2}(\gamma)(-\sin (\theta))\right) \\
& -x(\sin (2 \gamma) \sin (\theta) \sin (\psi) \\
& \left.\left.+\cos ^{2}(\gamma) \cos (\psi)-\sin ^{2}(\gamma) \cos (\psi)\right)\right), \\
& M_{44}=m_{2} \ell^{2} \cos ^{2}(\psi), M_{45}=0, M_{55}=m_{2} \ell^{2} \text {, }
\end{aligned}
$$


The gravitational term in the equations of motion is

$$
\mathbf{G}_{\text {non }}^{\boldsymbol{\top}}(\mathbf{q})=g m_{2}\left[\begin{array}{lllll}
0 & \cos (\theta) \cos (\psi) & 0 & -\ell \sin (\theta) \cos (\psi) & -\ell \cos (\theta) \sin (\psi)
\end{array}\right] .
$$

For brevity, full details of the nonlinear term $\mathbf{C}_{\text {non }}(\mathbf{q}, \dot{\mathbf{q}}) \dot{\mathbf{q}}$ are omitted, however, this term can be written as

$$
\mathbf{C}_{\mathrm{non}}(\mathbf{q}, \dot{\mathbf{q}}) \dot{\mathbf{q}}=-\dot{\mathbf{M}} \dot{\mathbf{q}}+\left(\frac{\partial}{\partial \mathbf{q}}\left(\frac{1}{2} \dot{\mathbf{q}}^{\top} \mathbf{M} \dot{\mathbf{q}}\right)\right)^{\top}
$$

